\title{
Erratum to: Exploitation of a tristable nonlinear oscillator for improving broadband vibration energy harvesting
}

\author{
Eur. Phys. J. Appl. Phys. (2014) 67: 30901
}

Shengxi Zhou, Junyi Cao ${ }^{\mathrm{a}}$, Jing Lin, and Zezhou Wang

State Key Laboratory for Manufacturing Systems Engineering, School of Mechanical Engineering, Xian Jiaotong University, Xian 710049, P.R. China

Received: 9 September 2014 / Accepted: 9 September 2014

Published online: 25 November 2014 - (c) EDP Sciences 2014

\begin{tabular}{ccc}
\hline Position & Source of error & Correction \\
\hline Equation (1) & Second "-" & Should be "+" \\
Equation (2) & "-" after $\dot{v}(t)$ & Should be "+" \\
Equation (3) & "-" after $\dot{v}(t)$ & Should be "+" \\
\hline
\end{tabular}

$$
\begin{gathered}
\left\{\begin{array}{l}
T=c^{E} S-e_{t} E \\
D=e S-\varepsilon^{S} E
\end{array}->\left\{\begin{array}{l}
T=c^{E} S-e_{t} E \\
D=e S+\varepsilon^{S} E
\end{array}\right.\right. \\
\left\{\begin{array}{l}
M \ddot{x}(t)+C \dot{x}(t)+K x(t)-\theta \mathrm{v}(t)=F \\
\dot{v}(t)-C_{p}^{-1} \theta \dot{x}(t)+C_{p}^{-1} R^{-1} v(t)=0
\end{array}->\left\{\begin{array}{l}
M \ddot{x}(t)+C \dot{x}(t)+K x(t)-\theta \mathrm{v}(t)=F \\
\dot{v}(t)+C_{p}^{-1} \theta \dot{x}(t)+C_{p}^{-1} R^{-1} v(t)=0
\end{array}\right.\right. \\
\left\{\begin{array}{l}
M \ddot{x}(t)+C \dot{x}(t)+f_{r}-\theta \mathrm{v}(t)=F \\
\dot{v}(t)-C_{p}^{-1} \theta \dot{x}(t)+C_{p}^{-1} R^{-1} v(t)=0
\end{array}\right.
\end{gathered}
$$

\footnotetext{
${ }^{a}$ e-mail: caojy@mail.xjtu.edu.cn
} 